Lecture Notes in Physics 457 (1995) 379-392, Springer-Verlag

\title{
Computer Simulation of Lévy $\alpha$-Stable Variables and Processes
}

\author{
Aleksander Weron * \\ and \\ Rafał Weron
}

The Hugo Steinhaus Center for Stochastic Methods, Technical University of Wroclaw, 50-370 Wrocław, Poland.

Abstract. The aim of this paper is to demonstrate how the appropriate numerical, statistical and computer techniques can be successfully applied to the modeling of some physical systems. We propose to use a fast and accurate method of computer generation of Lévy $\alpha$-stable random variates.

Keywords: $\alpha$-stable random variables and processes, Ornstein-Uhlenbeck process, continuous-time random walk, computer simulation, parameter estimators, tests for infinite variance.

\section{Introduction}

The past few years have witnessed an explosive growth in interest in physical and engineering systems that could be studied using stochastic and chaotic methods, see Berliner (1992), Chatterjee and Yilmaz (1992), and Shao and Nikias (1993). "Stochastic" and "chaotic" refer to nature's two paths to unpredictability, or uncertainty. To scientists and engineers the surprise was that chaos (making a very small change in the universe can lead to a very large change at some later time) is unrelated to randomness. Things are unpredictable if you look at the individual events; however, one can say a lot about averaged-out quantities. This is where the stochastic stuff comes in. Stochastic processes are recognized to play an important role in a wide range of problems encountered in mathematics, physics and engineering. Recent developments show that in many practical applications leading to appropriate stochastic models a particular class of Lévy $\alpha$-stable processes is involved. While the attempt at mathematical understanding of these processes leads to severe analytical difficulties, there exist very useful approximate numerical and statistical techniques (see Janicki and Weron (1994b)). Also non-Gaussian statistical methods in stochastic modeling are important when noises deviate from the ideal Gaussian model. Stable distributions are among the most important non-Gaussian models. They share defining characteristics with the Gaussian distribution, such as the stability property and central limit theorems, and include in fact the Gaussian

* Research of the first author was partially supported by the KBN Grant No.2 P302 06504. 
distributions as a special case. To help the interested reader better understand the stable models and necessary methodologies we discuss here tutorial examples of $\alpha-$ stable Ornstein-Uhlenbeck process and continuous-time random walks subject to $\alpha$-stable displacement.

\section{Computer Generation of Lévy $\alpha$-Stable Distri- butions}

The most common and convenient way to introduce Lévy $\alpha$-stable random variables is to define their characteristic function

$$
\log \phi(t)= \begin{cases}-\sigma^{\alpha}|t|^{\alpha}\left\{1-i \beta \operatorname{sign}(t) \tan \frac{\pi \alpha}{2}\right\}+i \mu t, & \alpha \neq 1, \\ -\sigma|t|\left\{1+i \beta \operatorname{sign}(t) \frac{2}{\pi} \log |t|\right\}+i \mu t, & \alpha=1,\end{cases}
$$

where $\alpha \in(0,2], \beta \in[-1,1], \sigma>0, \mu \in R$.

Since (2.1) is characterized by four parameters we will denote $\alpha$-stable distributions by $S_{\alpha}(\sigma, \beta, \mu)$ and write

$$
X \sim S_{\alpha}(\sigma, \beta, \mu)
$$

to indicate that $X$ has the stable distribution $S_{\alpha}(\sigma, \beta, \mu)$. When $\sigma=1$ and $\mu=0$ the distribution is called standard stable.

The location parameter $\mu$ shifts the distribution to the left or right. The scale parameter $\sigma$ compresses or extends the distribution about $\mu$. Some authors use $\gamma=\sigma^{\alpha}$ instead of $\sigma$, but it no longer has the natural interpretation of the scale parameter. The characteristic exponent $\alpha$ lies in the range $(0,2]$ and determines the rate at which the tails of the distribution taper off. When $\alpha=2$, a Gaussian distribution results, with mean $\mu$ and variance $2 \sigma^{2}$. When $\alpha<2$, the variance is infinite. When $\alpha>1$, the mean of the distribution exists and is equal to $\mu$. In general, the $p$-th moment of a stable random variable is finite iff $p<\alpha$. The fourth parameter, which determines the skewness of the distribution, is designated $\beta$ and lies in the range $[-1,1]$. When $\beta$ is positive, the distribution is skewed to the right. When it is negative, it is skewed to the left. When $\beta=0$, the distribution is symmetrical. As $\alpha$ approaches $2, \beta$ loses its effect and the distribution approaches the symmetrical Gaussian distribution regardless of $\beta$.

The complexity of the problem of simulation of sequences of stable random variables results from the fact that there are no analytic expressions for the inverse $F^{-1}$ of the distribution function. The only exceptions are the Gaussian $S_{2}(\sigma, 0, \mu)=N\left(\mu, 2 \sigma^{2}\right)$, Cauchy $S_{1}(\sigma, 0, \mu)$ and Lévy $S_{1 / 2}(\sigma, 1, \mu), S_{1 / 2}(\sigma,-1, \mu)$ distributions, for which simple methods of simulation have been found.

Even recently new ways for simulating stable random variables are sought for in physical literature. For example, Mantegna (1994) proposes a "fast and accurate" algorithm for the symmetric case $(\beta=0, \mu=0)$ based on Bergstrom series expansion. This algorithm may be fast but there are no proofs of its accuracy, except visual similarities of densities. Moreover, arguments used in the paper are not re-

liable since the author mixes the basic concepts of probability theory like density, distribution and stochastic process. 
We propose to use a well known in mathematical literature method of computer generation of a symmetric $\alpha$-stable random variable $X \sim S_{\alpha}(1,0,0)$. For $\alpha \in(0,2]$

- generate a random variable $V$ uniformly distributed on $\left(-\frac{\pi}{2}, \frac{\pi}{2}\right)$ and an independent exponential random variable $W$ with mean 1 ;

- compute

$$
X=\frac{\sin (\alpha V)}{(\cos (V))^{1 / \alpha}} \times\left(\frac{\cos (V-\alpha V)}{W}\right)^{(1-\alpha) / \alpha}
$$

In a similar way to (2.3), reparametrizing the algorithm of Chambers et al. (1976) or using the theorem of Weron (1995a), we can construct a method of computer generation of a skewed random variable $X \sim S_{\alpha}(1, \beta, 0)$. For $\alpha \in(0,2]$ and $\beta \in[-1,1]$

- generate a random variable $V$ uniformly distributed on $\left(-\frac{\pi}{2}, \frac{\pi}{2}\right)$ and an independent exponential random variable $W$ with mean 1 ;

- for $\alpha \neq 1$ compute

$$
X=S_{\alpha, \beta} \times \frac{\sin \left(\alpha\left(V+B_{\alpha, \beta}\right)\right)}{(\cos (V))^{1 / \alpha}} \times\left(\frac{\cos \left(V-\alpha\left(V+B_{\alpha, \beta}\right)\right)}{W}\right)^{(1-\alpha) / \alpha},
$$

where

$$
\begin{aligned}
& B_{\alpha, \beta}=\frac{\arctan \left(\beta \tan \frac{\pi \alpha}{2}\right)}{\alpha}, \\
& S_{\alpha, \beta}=\left[1+\beta^{2} \tan ^{2} \frac{\pi \alpha}{2}\right]^{1 /(2 \alpha)} ;
\end{aligned}
$$

- for $\alpha=1$ compute

$$
X=\frac{2}{\pi}\left[\left(\frac{\pi}{2}+\beta V\right) \tan V-\beta \log \left(\frac{\frac{\pi}{2} W \cos V}{\frac{\pi}{2}+\beta V}\right)\right] .
$$

Formula (2.4) was initially presented by Janicki and Weron (1994a). However, there is a misprint in the form for $C_{\alpha, \beta}$ (the denominator is $1-|1-\alpha|$ instead of $\alpha$, Formula (3.5.2), page 50), which corresponds to our $B_{\alpha, \beta}$, and a computationally more complicated form for $D_{\alpha, \beta}\left(\right.$ our $\left.S_{\alpha, \beta}\right)$.

We have given formulas for simulation of standard stable random variables. Using the following property, which follows from the form of the characteristic function, we can simulate a stable random variable for all admissable values of the parameters $\alpha, \sigma, \beta$ and $\mu$ :

If $X \sim S_{\alpha}(1, \beta, 0)$ then

$$
Y= \begin{cases}\sigma X+\mu, & \alpha \neq 1, \\ \sigma X+\frac{2}{\pi} \beta \sigma \log \sigma+\mu, & \alpha=1,\end{cases}
$$

is $S_{\alpha}(\sigma, \beta, \mu)$.

We regard the methods defined by (2.3), (2.4) and (2.5) as good techniques of computer generation of $\alpha$-stable random variables, stochastic measures and processes of different kinds. For more details see Janicki and Weron (1994a) and Weron (1995a). 


\section{Simulation of Lévy $\alpha$-stable stochastic processes}

Now we describe rather general technique of approximate computer simulation of univariate $\alpha$-stable stochastic processes $\{X(t): t \in[0, T]\}$ with independent increments, which is based on a construction of a discrete time process of the form $\left\{X_{t_{i}}^{\tau}\right\}_{i=0}^{I}$, defined by the formula

$$
X_{t_{i}}^{\tau}=X_{t_{i-1}}^{\tau}+\mathcal{F}\left(t_{i-1}, X_{t_{i-1}}^{\tau}\right)+Y_{i}^{\tau}
$$

with a given $X_{0}^{\tau}$, and where $Y_{i}^{\tau^{\prime}}$ s form a sequence of i.i.d. $\alpha$-stable random variables.

In computer calculations each random variable $X_{t_{i}}^{\tau}$ defined by $(3.1)$ is represented by its $\mathrm{N}$ independent realizations, i.e. a random sample $\left\{X_{i}^{\tau}(n)\right\}_{n=1}^{N}$. So, let us fix $N \in \mathcal{N}$ large enough. The algorithm consists in the following:

1. simulate a random sample $\left\{X_{0}^{\tau}(n)\right\}_{n=1}^{N}$ for $X_{0}^{\tau}$;

2. for $i=1,2, \ldots, I$ simulate a random sample $\left\{Y_{i}^{\tau}(n)\right\}_{n=1}^{N}$ for $\alpha$-stable random variable $Y_{i}^{\tau} \sim S_{\alpha}\left(\tau^{1 / \alpha}, 0,0\right)$, with appropriately chosen $\tau$;

3. for $i=1,2, \ldots, I$, in accordance with (3.1), compute the random sample $X_{i}^{\tau}(n)=X_{i-1}^{\tau}(n)+\mathcal{F}\left(t_{i-1}, X_{i-1}^{\tau}(n)\right)+Y_{i}^{\tau}(n), \quad n=1,2, \ldots, N$;

4. construct kernel density estimators $f_{i}=f_{i}^{I, N}=f_{i}^{I, N}(x)$ of the densities of $X\left(t_{i}\right)$, using for example the optimal version of the Rosenblatt-Parzen method.

Observe that we have produced $\mathrm{N}$ finite time series of the form $\left\{X_{i}^{\tau}(n)\right\}_{i=0}^{I}$ for $n=1,2, \ldots, N$. We regard them as "good" approximations of the trajectories of the process $\{X(t) ; t \in[0, T]\}$.

In particular, the above described algorithm can be succesfully applied to the construction of approximate solutions to the following linear stochastic differential equation driven by an $\alpha$-stable Lévy motion

$$
X(t)=X_{0}+\int_{0}^{t}(a(s)+b(s) X(s-)) d s+\int_{0}^{t} c(s) d L_{\alpha}(s) \quad \text { for } \quad t \in[0, \infty),
$$

with $X(0)=X_{0}$ a given $\alpha$-stable or discrete random variable.

Let us notice that this linear stochastic equation is of independent interest because, as is easily seen, the general solution belongs to the class of $\alpha$-stable processes. It may be expressed in the following form

$$
X(t)=\Phi(t, 0) X_{0}+\int_{0}^{t} \Phi(t, s) a(s) d s+\int_{0}^{t} \Phi(t, s) c(s) d L_{\alpha}(s)
$$

where $\Phi(t, s)=\exp \left\{\int_{s}^{t} b(u) d u\right\}$.

This explains why outliers or heavy tails appear in the constructed approximate solutions $\left\{X_{i}^{\tau}(n)\right\}_{i=0}^{I}, n=1,2, \ldots, N$, to $(3.2)$, which can be directly derived as a special case of (3.1). It is enough to define the set $\left\{t_{i}=i \tau, i=0,1, \ldots, I\right\}, \tau=T / I$, describing a fixed mesh on the interval $[0, T]$, and the sequence of i.i.d. random variables $\Delta L_{\alpha, i}^{\tau}$ playing the role of the random $\alpha$-stable measure of the interval $\left[t_{i-1}, t_{i}\right)$, i.e. an $\alpha$-stable random variable defined by

$$
\Delta L_{\alpha, i}^{\tau}=L_{\alpha}\left(\left[t_{i-1}, t_{i}\right)\right) \sim S_{\alpha}\left(\tau^{1 / \alpha}, 0,0\right)
$$


and to choose $X_{0}^{\tau}=X_{0} \sim S_{\alpha}(\sigma, 0, \mu)$, computing

$$
X_{t_{i}}^{\tau}=X_{t_{i-1}}^{\tau}+\left(a\left(t_{i-1}\right)+b\left(t_{i-1}\right) X_{t_{i-1}}^{\tau}\right) \tau+c\left(t_{i-1}\right) \Delta L_{\alpha, i}^{\tau},
$$

for $i=1,2, \ldots, I$.

An appropriate convergence result justifying the method can be found in Janicki, Michna and Weron (1994).

In order to obtain a graphical computer presentation of the discrete time stochastic process of the form (3.1), we propose the following approach:

1. fix a rectangle $[0, T] \times[c, d]$ that should include the trajectories of $\{X(t)\}$;

2. for each $n=1,2, \ldots, n_{\max }$ (with fixed $n_{\max } \ll N$ ) draw the line segments determined by the points $\left(t_{i-1}, X_{i-1}^{\tau}(n)\right)$ and $\left(t_{i}, X_{i}^{\tau}(n)\right)$ for $i=1,2, \ldots, I$, constructing $n_{\max }$ approximate trajectories of the process $X$;

3. fixing values of a parameter $p_{j} \in(0,1), j=1,2, \ldots, J$, it is possible do derive from each statistical sample $\left\{X_{i}^{\tau}(n)\right\}_{n=1}^{N}$ with fixed $i \in\{0,1, \ldots, I\}$ estimators of corresponding quantiles $q^{i, j}=F_{i}^{-1}\left(p_{j}\right)$, where $F_{i}=F_{i}(x)$ denotes the unknown density distribution function of the random variable $X_{t_{i}}^{\tau}$ represented by the statistical sample $\left\{X_{i}^{\tau}(n)\right\}_{n=1}^{N}$. In this way we obtain approximation of the, so called, quantile lines, i.e., the curves $q_{j}=q_{j}(t)$ defined by the condition $P\left\{X(t) \geq q_{j}(t)\right\}=p_{j}$.

\subsection{Construction of an $\alpha$-stable Ornstein-Uhlenbeck pro- cess}

To describe a motion of a particle in a fluid in a model with infinite variance (see, e.g., West and Seshadri (1982)) we have to consider a random strength. In the classical Langevin equation we obtain that this strength is Gaussian and this equation has a form

$$
V(t)=V(0)-\lambda \int_{0}^{t} V(s) d s+\mu \int_{0}^{t} d B(s)
$$

where $V$ is a velocity of the particle and $-\lambda \int_{0}^{t} V(s) d s$ describes the strength of a resistance of an environment according to Stokes law and $\mu \int_{0}^{t} d B(s)$ is a random strength ( $\mu$ and $\lambda$ denoting some given constants).

Generalizing the model we can consider an $\alpha$-stable random strength and we can rewrite the previous equation as follows

$$
V(t)=V(0)-\lambda \int_{0}^{t} V(s) d s+\mu \int_{0}^{t} d L_{\alpha}(s)
$$

where $\{\mathbf{L}\}_{\alpha}=\{L(t)\}$ is an $\alpha$-stable Lévy motion.

Applying the result of Janicki, Michna and Weron (1994) with some obvious modifications we obtain the process $\mathbf{V}_{n}$ approximating the process $\mathbf{V}$. This leads to the following formula

$$
V_{n}(t)=V(0) \exp (-\lambda t)+\mu \int_{0}^{t} \exp (-\lambda(t-s)) d L_{\alpha}^{(n)}(s)
$$




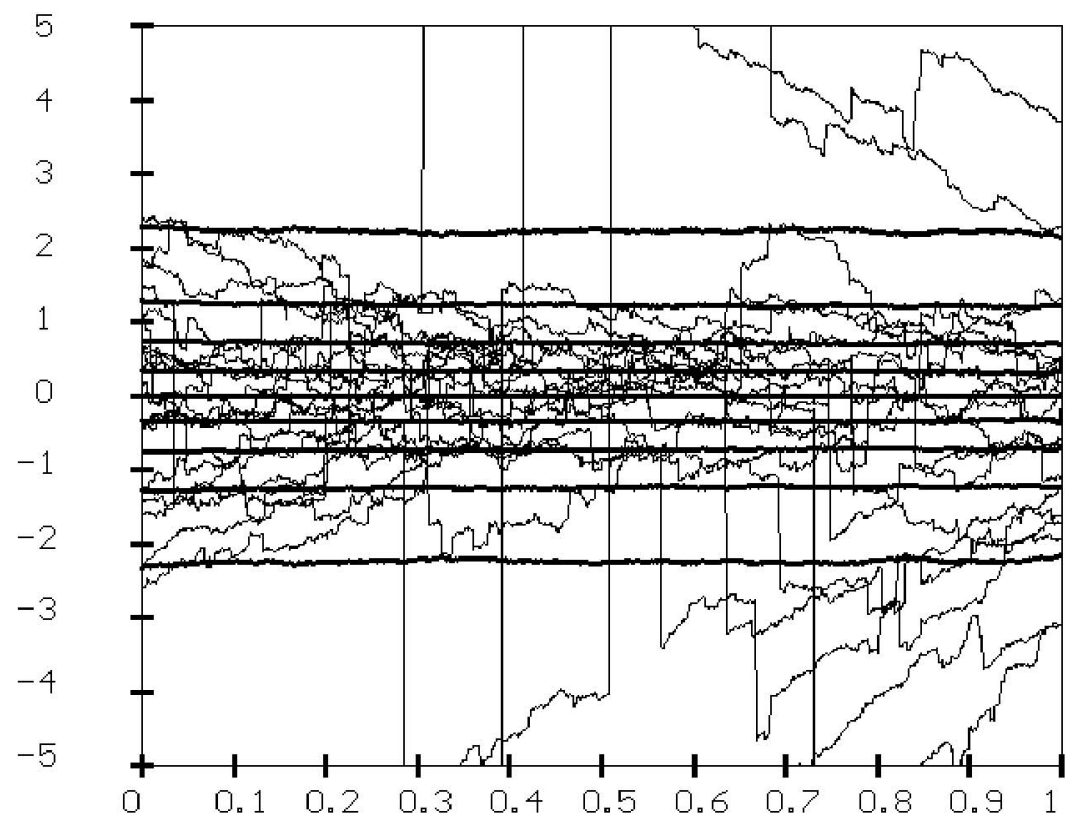

Figure 3.1: Solution to (3.6) in the case of $\lambda=2.0$ and $\mu=2.0$.

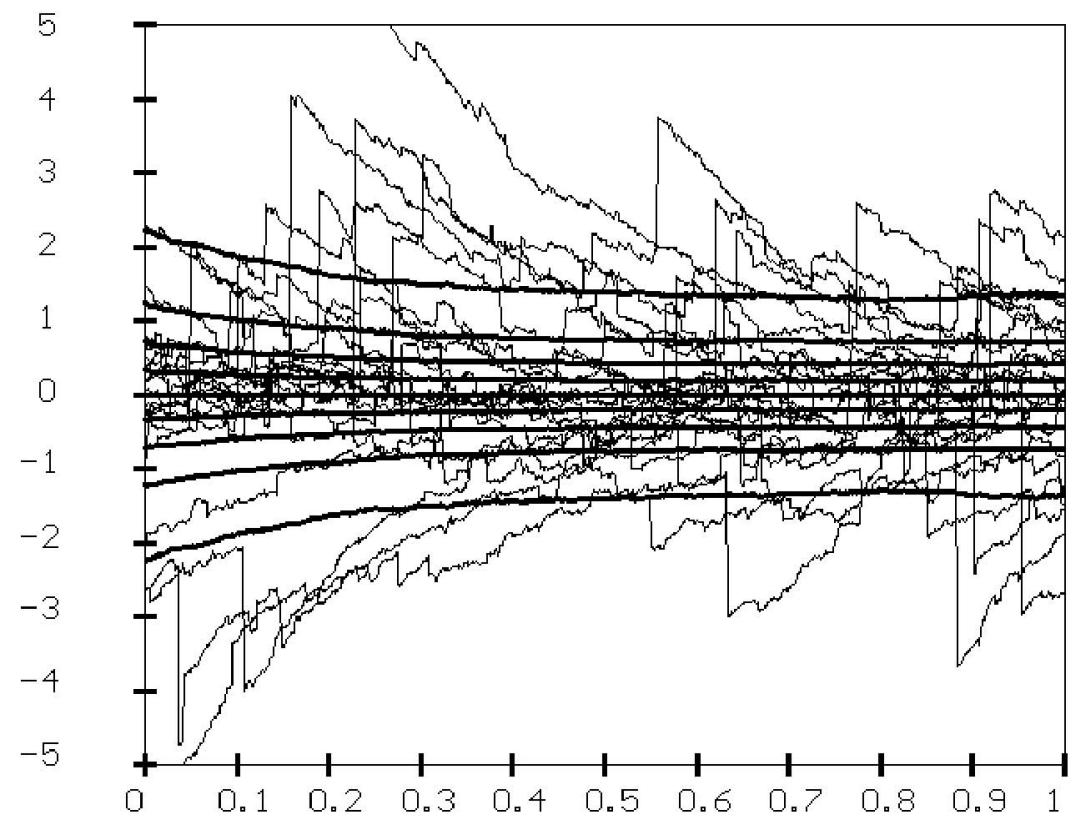

Figure 3.2: Solution to (3.6) in the case of $\lambda=4.0$ and $\mu=2.0$. 
where $L_{\alpha}^{(n)}(t)=\sum_{j=1}^{[n t]} \frac{Y_{j}}{\phi(n)}$, and $\sum_{j=1}^{n} \frac{Y_{j}}{\phi(n)} \stackrel{\mathcal{L}}{\rightarrow} L_{\alpha}(1)$.

Fig. 3.1 - 3.2 contain graphical results of computer simulations of solutions to equation (3.6) for $\alpha=1.3$. The problem was to find a value for $\lambda$ assuring stationarity of the process $\{V(t): t \geq 0\}$, provided $V(0) \sim S_{1.3}(1,0,0)$ and $\mu=2.0$ were fixed. The figures present the results obtained for two different values of $\lambda=$ 2.0, 4.0. It follows from the presented computer experiment that the proper value of $\lambda$ should be chosen close to 2.0. This can be read from the shape of quantile lines, because horizontal quantile lines characterize stationary processes.

\section{Tests for Infinite Variance}

In some physical applications, arises the question whether the stable distribution is Gaussian or non-Gaussian. There exist simple and computationally convenient methods that are used in practice. The handicap of all three mentioned here methods is that they rely on visual inspection.

\subsection{Converging Variance Test}

Given a sample $X_{1}, \ldots, X_{n}$ from a stable distribution, for each $1 \leq i \leq n$ form a statistics based on the first $i$ observations

$$
S_{i}^{2}=\frac{1}{i-1} \sum_{k=1}^{i}\left(X_{k}-\bar{X}_{i}\right)^{2}
$$

where $\bar{X}_{i}=\frac{1}{i} \sum_{k=1}^{i} X_{i}$. Then, plot $S_{i}^{2}$ against $i$. If the population distribution has a finite variance, $S_{i}^{2}$ should converge to a finite value. Otherwise, $S_{i}^{2}$ should diverge. Distinguishing between these two cases is rather vague, especially when we have only one trajectory (one sample).

We have checked this method on four samples of size 4000 coming from $S_{\alpha}(1,0,0)$, for four different $\alpha$ 's. Fig. 4.1 presents the results. For $\alpha=2$, the distribution is Gaussian with mean $\mu=0$ and a finite variance $\sigma^{2}=2$. As well known, in this case $E S_{i}^{2}=\sigma^{2}=2$ and $\operatorname{Var} S_{i}^{2}=\frac{2}{i-1} \sigma^{4}=\frac{8}{i-1}$. When we took smaller $\alpha$, the plot became more rugged and the values of $S_{i}^{2}$ were much larger (note the change of scale on plots (c) and (d)), which suggested infinite variance of the population distribution.

It is much easier to distinguish between finite and infinite variance when we have a few samples from each distribution. Fig. 4.2 shows five trajectories for every $\alpha=2.0,1.95,1.5,0.5$. In the latter case, the largest jumps in the trajectories differed by an order of magnitude and it was difficult to find two paths that would be visible on the same scale.

\section{$4.2 \quad \mathrm{Q}-\mathrm{Q}$ Plot}

Q-Q plots (or probability plots) are widely used since they provide quick estimates and a quick informal assessment about the fit of a distribution. Furthermore, when a distribution does not fit, the plot usually tells why this is the case, for instance 


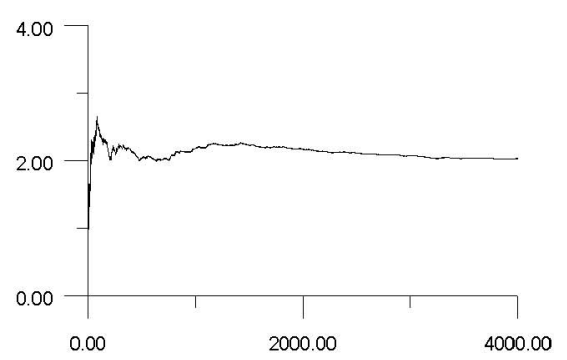

(a)

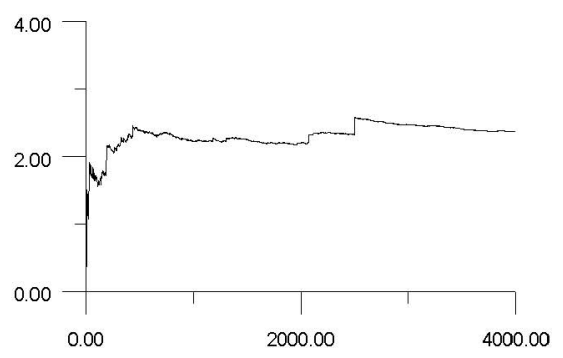

(b)

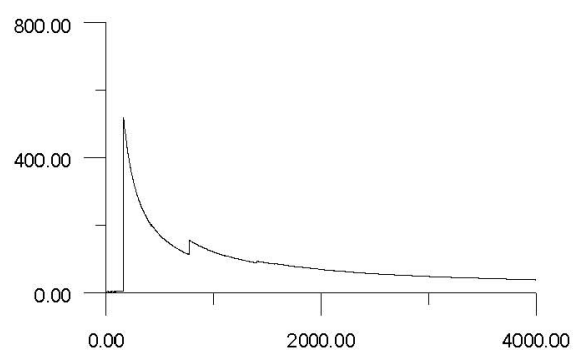

(c)

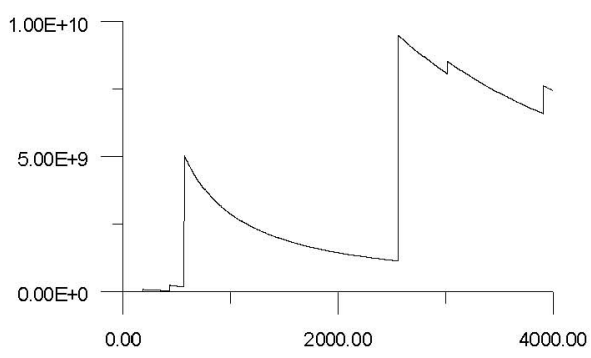

(d)

Figure 4.1: Plots of the statistics $S_{i}^{2}$ against $i=1, \ldots, 4000$ for $S_{\alpha}(1,0,0)$ with (a) $\alpha=2.0$; (b) $\alpha=1.95$; (c) $\alpha=1.5$; (d) $\alpha=0.5$.

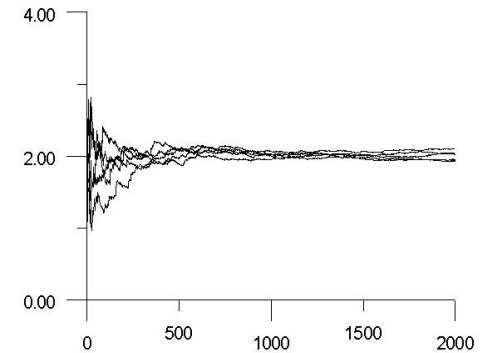

(a)

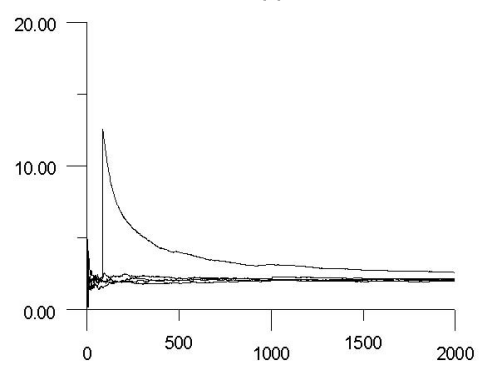

(b)

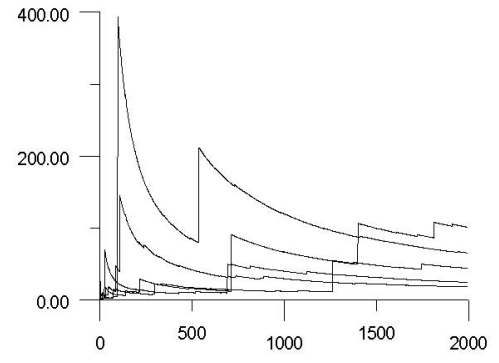

(c)

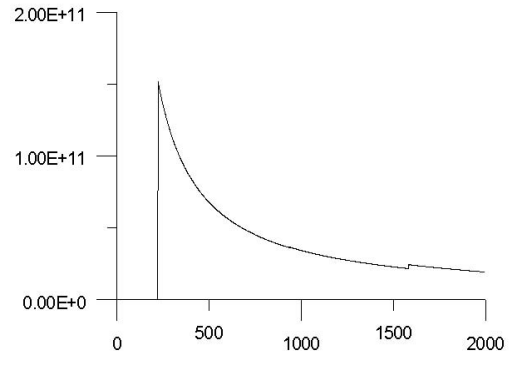

(d)

Figure 4.2: Plots of five trajectories of the statistics $S_{i}^{2}$ against $i=1, \ldots, 2000$ for $S_{\alpha}(1,0,0)$ with (a) $\alpha=2.0$; (b) $\alpha=1.95$; (c) $\alpha=1.5$; (d) $\alpha=0.5$. 
is it because of a single outlying observation or because of a systematic departure from the assumptions.

If we want to test whether certain data $X_{1}, \ldots, X_{n}$ follow the distribution function $F$, we plot the data on $F$-probability paper. This is based on a transformation of the distribution function $F$ (in this case Gaussian) into a straight line by applying $F$ to the vertical scale. The graph of $y=F(x)$ against $x$ is a straight line $y=x$.

The theoretical basis of plotting on probability paper (see Daniel (1976) or Embrechts et al. (1995)) is the fact that for $F$ continuous the random variable $F\left(X_{1}\right)$ is uniform on $(0,1)$. Hence, for the ordered sample $X_{(1)} \leq \ldots \leq X_{(n)}$ we know that

$$
E\left[F\left(X_{(k)}\right)\right]=\frac{k}{n+1}, \quad k=1, \ldots, n .
$$

Consequently, we could plot $\frac{k}{n+1}$ against $F\left(X_{(k)}\right)$. However, more common is to plot $X_{(k)}$ against $F^{-1}\left(\frac{k}{n+1}\right)$.

If the plot is not approximately linear, the population from which the data seemed to be drawn was not $F$. In our case this means that the data probably came from a distribution with infinite variance, see Daniel (1976).

\subsection{Log-Tail Test}

This test examines the shape of the tails of the estimated distribution. Mandelbrot (1963) suggested that because a non-Gaussian stable variable satisfies the relation

$$
\lim _{x \rightarrow \infty} x^{\alpha} P(X>x)=\text { const, }
$$

then for large $x$ a plot of $\log P(X>x)$ against $\log x$ should yield a straight line with slope $-\alpha$. We can use the sample distribution function

$$
F_{n}(x)=\frac{1}{n} \#\left\{i: x_{i} \leq x\right\},
$$

to estimate $P(X \leq x)=1-P(X>x)$.

We have tested this method on samples of size 10000 coming from $S_{\alpha}(1,0,0)$, for four different $\alpha$ 's. For $\alpha=2.0$ the distribution is Gaussian and has exponential (not inverse power !) tails (see Samorodnitsky and Taqqu (1994), page 16). Therefore, the plot is not a straight line. For smaller $\alpha$, the plot is approximately a straight line and the slope is a rough estimate of $-\alpha$. An obvious disadvantage of this method is the size of the sample needed. For small sizes $(n<1000)$ the plot becomes rugged and it is difficult to find the slope. We discourage from using this approach for small samples. It also gives only a poor estimate of $\alpha$. Especially for $\alpha>1.5$, as the tails of the distribution become smaller, our estimate is subject to a large error, since the plot significantly deviates from a straight line. See Fig. 4.3.

\section{Continuous-Time Random Walk}

Continuous-Time Random Walk (CTRW) is a stochastic process with random waiting times $T_{i}$ between successive jumps of random length $R_{i}$. During the recent years 


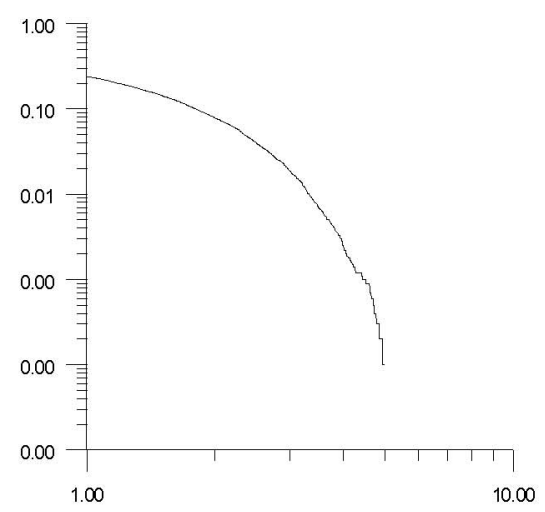

(a)

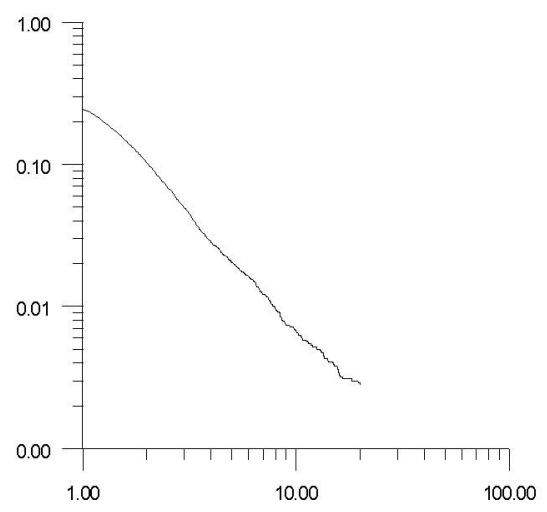

(b)

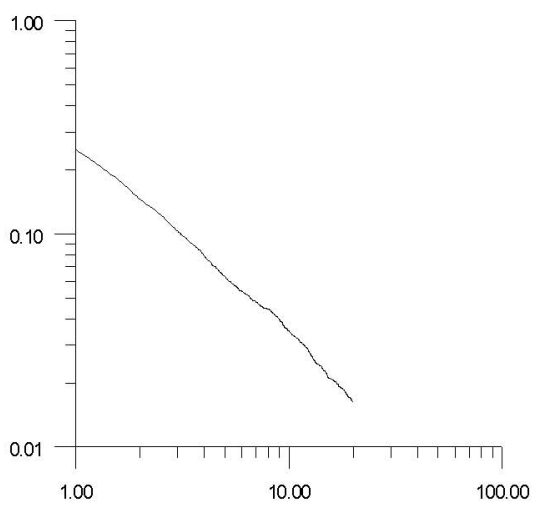

(c)

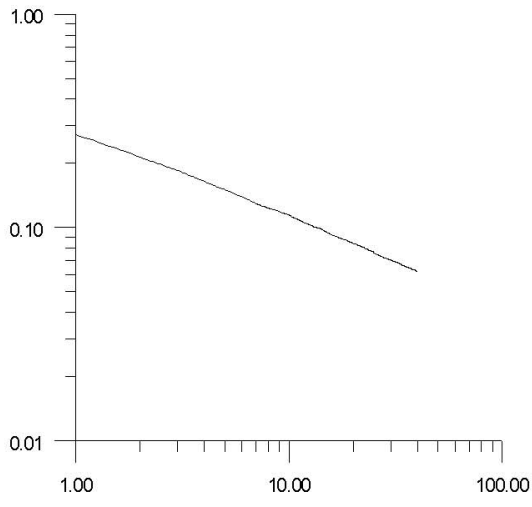

(d)

Figure 4.3: Log-Tail test: plots of $\log \left(1-F_{n}(x)\right)$ against $\log x$ for $S_{\alpha}(1,0,0)$ with (a) $\alpha=2.0$; (b) $\alpha=1.5$; (c) $\alpha=1.0$; (d) $\alpha=0.5$.

it has been studied extensively and applied to turbulence, transport in disordered or fractal media, intermittent chaotic systems and relaxation phenomena. For references see Klafter et al. (1993). The common feature of these applications is that they exhibit anomalous diffusion, which is manifested through non-linear time dependence of a mean square distance $R_{t}$ reached by a particle up to the moment $t$ (with the initial condition $R_{0}=0$ ).

For simplicity we restrict ourselves to one-dimensional walks. The first instantaneous jump of random length $R_{1}$ is executed after a random waiting time $T_{1}$. Then the second instantaneous jump $R_{2}$ (i.e. jump of random length $R_{2}$ ) is executed after time $T_{2}$, etc. In general, the $i$-th jump $R_{i}$ is dependent on its waiting time $T_{i}$, but the pairs $\left(R_{i}, T_{i}\right)$ are independent for different $i$ 's. See Fig. 5.1. The special case when $R_{i}$ is independent of $T_{i}$ is called the decoupled memory CTRW as opposed to the coupled one with $R_{i}$ dependent on $T_{i}$. If we define the random variable $N_{t}$ as the number of jumps in the time interval $[0, t]$

$$
N_{t}=\max \left\{k: \sum_{i=0}^{k} T_{i} \leq t\right\} \text {, }
$$


then it is clear that the position $R_{t}$ of the particle at the time $t$ is equal to a random sum of $N_{t}$ successive random jumps $R_{i}$, i.e.

$$
R_{t}=\sum_{i=0}^{N_{t}} R_{i}
$$

Note, that (5.2) holds also for a random walk when waiting time intervals $T_{i}$ are non-random and take a constant value.

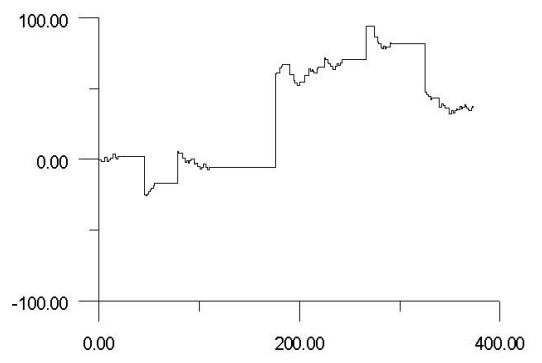

(a)

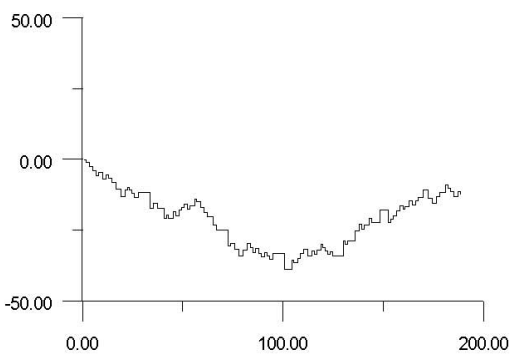

(b)

Figure 5.1: Two sample paths of a coupled memory CTRW: plots of the distance $R_{t}$ against time with (a) $\alpha=1.2$ and (b) $\alpha=1.8$. There are 100 jumps in the trajectories on both plots. Note the scale difference on both axes.

Many authors were interested in finding the asymptotic distribution of the position $R_{t}$ of a particle at time $t$. We concentrated our research on the following formula

$$
\lim _{t \rightarrow \infty}\left(\frac{R_{t}}{(t / \tau)^{1 / \alpha} c_{2}}<x\right)=S_{\alpha}(1,0,0)(x),
$$

which is valid for $1<\alpha<2$ and $\tau=E T_{i}$. $T_{i}$ has a density $g(t)$ such that $\lim _{t \rightarrow \infty} \frac{g(t)}{\alpha t^{-\alpha-1}}=1$, and $R_{i}$ satisfies $P\left(R_{i}=T_{i}\right)=P\left(R_{i}=-T_{i}\right)=\frac{1}{2}$. The normalizing constant in (5.3) is given by $c_{2}=c_{\alpha}^{-1 / \alpha}$, where

$$
c_{\alpha}=\frac{1-\alpha}{\Gamma(2-\alpha) \cos \left(\frac{\pi \alpha}{2}\right)},
$$

and $\Gamma$ denotes the Euler Gamma function. This is a coupled memory CTRW. Fig. 5.1 presents two sample paths, with 100 jumps each, for two values of $\alpha$. For $\alpha=1.2$ the distribution of $T_{i}$, and thus of $R_{i}$, has longer tails then for $\alpha=1.8$. This results in longer waiting times and jumps. A trajectory for $\alpha=1.2$ would, in general, make twice less jumps in the same time then a trajectory for $\alpha=1.8$.

In our simulations, we let $T_{i}$ have a $\operatorname{Pareto}(\alpha, 1)$ distribution, i.e. $g(t)=\alpha t^{-\alpha-1}$. A random variable $X$ having this distribution can be easily constructed using the inverse transform method

- generate a random variable $\mathrm{V}$ distributed uniformly on $(0,1)$;

- compute $X=V^{-1 / \alpha}$. 
We have chosen three values of $\alpha: 1.2,1.5$ and 1.8 in the permissible range of $(1,2)$. For every $\alpha$ we ran 25 simulations with 2000 trajectories each. Then for every $\alpha$ and every stopping time $t=500,1000,5000$ we used the regression method of Koutrouvelis (1980), which is the most reliable among the known statistical methods (see Weron (1995b)), to estimate $\alpha$ and $\sigma$ (the distribution of $R_{t}$ is symmetric). The results are summarized in Table 5.1.

\begin{tabular}{|c|r|rrrrrrrr|}
\hline$\alpha$ & $t$ & $\bar{\alpha}$ & $\alpha_{\min }$ & $\alpha_{\max }$ & $M S E_{\alpha}$ & $\bar{\sigma}$ & $\sigma_{\min }$ & $\sigma_{\max }$ & $M S E_{\sigma}$ \\
\hline \hline 1.2 & 500 & 1.312 & 1.237 & 1.402 & 0.0139 & 1.629 & 1.530 & 1.739 & 0.3979 \\
\hline 1.2 & 1000 & 1.292 & 1.220 & 1.352 & 0.0101 & 1.530 & 1.444 & 1.639 & 0.2836 \\
\hline 1.2 & 5000 & 1.260 & 1.206 & 1.337 & 0.0047 & 1.364 & 1.321 & 1.412 & 0.1330 \\
\hline \hline 1.5 & 500 & 1.539 & 1.450 & 1.623 & 0.0033 & 1.081 & 1.020 & 1.131 & 0.0073 \\
\hline 1.5 & 1000 & 1.519 & 1.436 & 1.601 & 0.0016 & 1.064 & 1.011 & 1.121 & 0.0048 \\
\hline 1.5 & 5000 & 1.513 & 1.464 & 1.586 & 0.0013 & 1.037 & 0.952 & 1.082 & 0.0022 \\
\hline \hline 1.8 & 500 & 1.727 & 1.665 & 1.790 & 0.0064 & 0.807 & 0.781 & 0.835 & 0.0374 \\
\hline 1.8 & 1000 & 1.735 & 1.642 & 1.796 & 0.0058 & 0.823 & 0.791 & 0.859 & 0.0318 \\
\hline 1.8 & 5000 & 1.746 & 1.682 & 1.814 & 0.0040 & 0.840 & 0.819 & 0.871 & 0.0259 \\
\hline
\end{tabular}

Table 5.1: Parameter estimates using the regression method of $\alpha=1.2,1.5,1.8$ and $\sigma$ describing the stable distribution of $R_{t}$ at stopping times $t=500,1000,5000$.

The distribution of $R_{t}$ is symmetric, i.e. $\beta=0, \mu=0$, thus we estimated only $\alpha$ and $\sigma$. For each parameter we give the mean, minimum, maximum and Mean Squarred Error i.e.

$$
M S E_{\theta}=\frac{1}{n} \sum_{i=1}^{r}\left(\hat{\theta}_{i}-\theta\right)^{2},
$$

of the estimates $\hat{\alpha}_{k}$ and $\hat{\sigma}_{k}$ obtained in 25 simulations $(k=1, \ldots, 25)$.

As we have stated before, the smaller $\alpha$ the larger are the waiting times and jumps, which is a consequence of longer tails of the distribution of $T_{i}$. This behavior can be observed in Table 5.1. The stopping time $t=500$ for $\alpha=1.8$ is more or less equivalent to the stopping time $t=1000$ for $\alpha=1.2$. This is the reason the convergence, to the population values of $\alpha$ and $\sigma$, for $\alpha=1.8$ seems to be slower than for $\alpha=1.2$. Moreover, for $\alpha=1.2$ the true values are overestimated, for $\alpha=1.8$ underestimated and for $\alpha=1.5$ the estimators give almost a perfect match. This, in fact, confirms what other authors have recently observed for different tail thickness (they have only estimated the parameter $\alpha$ ) of the distribution of $T_{i}$, see e.g. Zumofen et al. (1989). As $\alpha$ approaches 2 from below or 1 from above the convergence becomes very slow.

\section{References}

L. M. Berliner (1992), Statistics, probability and chaos, Stat. Science 7, 69-90.

S. Chatterjee and M. R. Yilmaz (1992), Chaos, fractals and statistics, Stat. Science $7,49-68$.

J. M. Chambers, C. L. Mallows and B. Stuck (1976), A method for simulating stable random variables, J. Amer. Statist. Assoc. 71, 340-344.

C. Daniel (1976). Applications of Statistics to Industrial Experimentation, Wiley \& Sons, New York.

L. Devroye (1987), A Course in Density Estimation, Birkhäuser, Boston. 
P. Embrechts, C. Kluppelberg and T. Mikosch (1995). Modelling Extremal Events in Insurance and Finance, in preparation.

A. Janicki, Z. Michna and A. Weron (1994), Approximation of stochastic differential equations driven by $\alpha$-stable Lévy motion, preprint.

A. Janicki and A. Weron (1994a), Simulation and Chaotic Behavior of $\alpha$-Stable Stochastic Processes, Marcel Dekker, New York.

A. Janicki and A. Weron (1994b), Can one see $\alpha$-stable variables and processes?, Stat. Science, 9, 109-126.

M. Kanter (1975), Stable densities under change of scale and total variation inequalities, Ann. Probab. 31, 697-707.

J. Klafter, G. Zumofen and M.F. Shlesinger (1993), Fractal description of anomalous diffusion in dynamical systems, Fractals, 1, 389-404.

I.A. Koutrouvelis (1980). Regression-type estimation of the parameters of stable laws, J. Amer. Statist. Assoc., 75, 918-928.

A. Lasota and M. C. Mackey (1994), Chaos, Fractals, and Noise. Stochastic Aspects of Dynamics, Springer, New York.

B. Mandelbrot (1963). The variation of certain speculative prices, J. Business, 36, 394-419.

B. Mandelbrot and J. W. Van Ness (1968), Fractional Brownian motions, fractional noises and applications, SIAM Review, 10, 422-437.

R.M. Mantegna (1994), Fast, accurate algorithm for numerical simulation of Lévy stable stochastic process, Phys. Rev. E, 49 4677-4683.

G. Samorodnitsky and M.S. Taqqu (1994). Stable Non-Gaussian Random Processes, Chapman \& Hall, New York.

M. Shao and C. L. Nikias (1993), Signal processing with fractional lower order moments: stable processes and their applications, Proc. IEEE, 81, 986-1010.

A. Weron (1984), Stable processes and measures: A survey, in Probability Theory on Vector Spaces III (D. Szynal, A. Weron, eds.) 306-364, Lecture Notes in Mathematics 1080, Springer, New York.

R. Weron (1995a), On the Chambers-Mallows-Stuck method for simulating skewed stable random variables, Statist. Probab. Lett., submitted.

R. Weron (1995b), Performance of the estimators of stable law parameters, Appl. Math., submitted.

B.J. West and V. Seshadri (1982), Linear systems with Lévy fluctuations, Physica A 113, 203-216.

G. Zumofen, A. Blumen, J. Klafter and M.F. Schlesinger (1989), Lévy walks for turbulence: A numerical study, J. Stat. Phys., 54, 1519-1528. 